\title{
Large area electron beam annealing
}

\author{
Cameron A. Moore, J. J. Rocca, T. Johnson, and G. J. Collins \\ Colorado State University, Fort Collins, Colorado 80523 \\ P. E. Russella) \\ Solar Energy Research Institute, 1536 Cole Boulevard, Golden, Colorado 80401
}

(Received 25 April 1983; accepted for publication 25 May 1983)

\begin{abstract}
We have achieved wide area $\left(38 \mathrm{~cm}^{2}\right)$ electron beam annealing of ion implanted silicon wafers using a glow discharge electron beam with electron energies between 3 and $7 \mathrm{keV}$. A continuous beam $7 \mathrm{~cm}$ in diameter with a power density up to $90 \mathrm{~W} / \mathrm{cm}^{2}$ was used to anneal the 7 -cm-diam central portion of boron-implanted $\left(30 \mathrm{keV}, 5 \times 10^{15}\right.$ atoms $\left./ \mathrm{cm}^{2}\right) n$-type $\langle 100\rangle$ silicon wafers 10 $\mathrm{cm}$ in diameter. Annealing was obtained without redistribution of the original dopant profile using a 15 -s electron beam exposure. Due to the high electron beam power density achieved over a large area, one can uniformly anneal an entire wafer in a single exposure without sample or beam scanning.
\end{abstract}

PACS numbers: $81.40 . \mathrm{Ef}, 41.80 . \mathrm{Dd}, 61.70 . \mathrm{Wp}, 52.80 . \mathrm{Hc}$

Annealing of ion implantation damage in semiconductor substrates has been accomplished using a variety of transient heating sources including pulsed and continuous lasers, arc lamps, and graphite strip heaters. ' Annealing via electron beams has also been achieved using point, ${ }^{2}$ line, ${ }^{3}$ and broad area sources. ${ }^{4}$ The previous electron beam annealing work ${ }^{2,3}$ employed high voltage ( $>20 \mathrm{kV}$ ) beams of small area that were focused to reach the required energy density. The broad area source used previously ${ }^{4}$ was a high voltage $(20$ $100 \mathrm{kV})$ high current (10-50 kA) field emission discharge that provides a 100 -ns pulsed electron beam. In annealing applications it caused liquid phase regrowth of the implant damaged layer of the semiconductor substrate thereby causing undesired redistribution of the as-implanted dopant profile. ${ }^{4}$ More recently, an electron beam generated by a glow discharge was used to anneal ion implanted silicon. ${ }^{5}$ In this experiment a 10-cm-diam aluminum cathode operating in pure helium produced a maximum discharge current of 80 $\mathrm{mA}$ at $9 \mathrm{kV}$ (unfocused electron beam power density was $\sim 9$ $\mathrm{W} / \mathrm{cm}^{2}$ ). To achieve the necessary power density for annealing silicon the electron beam was focused to a $2-\mathrm{cm}$ diameter using a magnetic lens. To anneal samples larger than this would require the beam to be scanned across the $\mathrm{Si}$ wafer.

In this letter we describe a high power density ( $>90 \mathrm{~W} /$ $\mathrm{cm}^{2}$ unfocused), broad area, and continuous electron source capable of annealing silicon wafers without the need of beam focusing or scanning. The broad area electron beam source with $90 \mathrm{~W} / \mathrm{cm}^{2}$ unfocused beam power is produced by a glow discharge electron gun operating in helium at pressures between 0.3 and 2 Torr. That is, electron beam generation occurs without the need of high vacuum. The large area, high power density and 1 Torr pressure beam generation are considerable practical advantages as compared to previous sources. ${ }^{2-4}$ Our beam source possesses one order of magnitude larger electron beam power densities than those previously reported by Ianno et al. ${ }^{5}$

Figure 1 shows the electron gun cathode in the bottom

${ }^{a)}$ Current address: JEOL USA, 11 Dearborn Rd., Peabody, MA 01960. of the photograph, the electron beam substained negative glow in the middle, and a 10-cm-diam silicon wafer heated by the beam electrons at the top. As can be seen from the thermal radiation, only the $7-\mathrm{cm}$ central portion of the $10-$ $\mathrm{cm}$-diam wafer is heated by the electron beam in the present work which employs a 7-cm-diam electron gun cathode.

The broad area glow discharge electron beam generation has been described in detail elsewhere. ${ }^{6}$ The cathode is made from a new binary compound that enhances the secondary electron emission coefficient $\gamma$, by a factor of 10 from that of pure metal electrodes. ${ }^{5}$ Equal portions (by weight) of molybdenum and magnesium oxide particles $10 \mu \mathrm{m}$ in diameter were cosintered to form the cathode. The $\mathrm{MgO}$ has a high secondary emission coefficient by ion bombardment allowing for efficient production of beam electrons while the Mo enables dc operation of the electron beam because the sintered cathode material is still a good conductor of electricity. This cathode, in contrast to those made from oxidecoated aluminum and oxide-coated magnesium described in Ref. 6, can operate at a high discharge current densities in a pure noble gas atmosphere without degradation of the electron beam generation efficiency. In this work, the electron

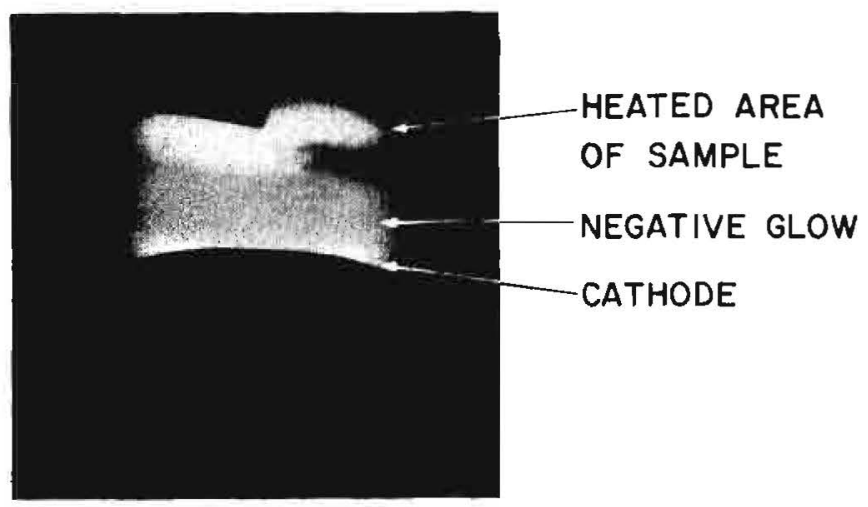

FIG. 1. Large area glow discharge electron beam annealing Si wafer. The cathode, the negative glow, and heated central area of the wafer are indicated. 


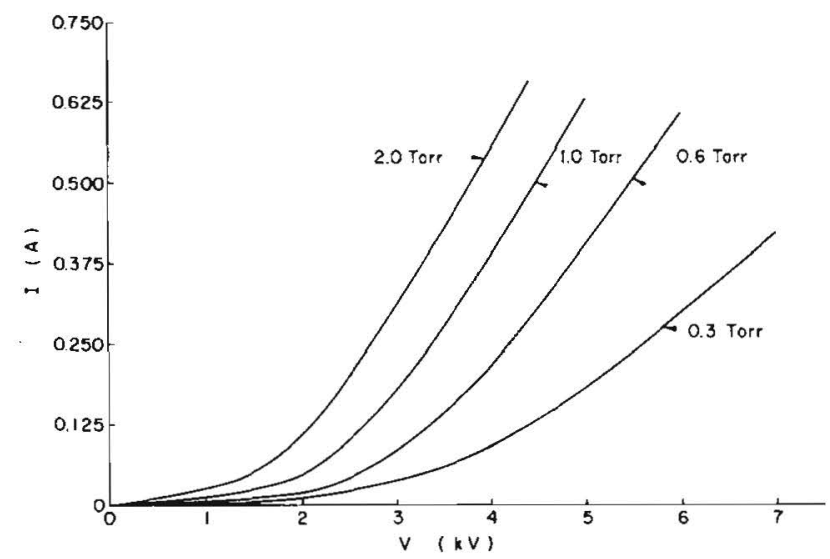

FlG. 2. Current-voltage characteristics of the glow discharge electron beam for $0.3,0.6,1.0$, and 2.0 Torr helium pressures.

gun is surrounded by a quartz shield that confines emission to only the front face of the cathode whose geometry results in a circular electron beam $7 \mathrm{~cm}$ in diameter.

The current-voltage characteristics of the electron beam glow discharge produced with the sintered cathode material at helium pressures of $0.3,0.6,1.0$, and 2.0 Torr are shown in Fig. 2. Discharge currents up to $0.8 \mathrm{~A}$ and discharge powers of $3.5 \mathrm{~kW}\left(\sim 90 \mathrm{~W} / \mathrm{cm}^{2}\right)$ were obtained. We have also successfully operated cathodes made of sintered mixtures of aluminum oxide and molybdenum, obtaining similar electron beam generation characteristics.

Application of this broad area electron source to single exposure annealing of ion implant damage in silicon substrates, without beam scanning, was examined. Wafers of 〈100) $n$-type silicon were implanted with $30-\mathrm{keV}$ ions of boron to a total dose of $5 \times 10^{15}$ ions $/ \mathrm{cm}^{2}$. Implanted samples had a measured preanneal sheet resistivity of $800 \pm 150$ $\Omega / \square$. Boron implantation was chosen because of its high solid state diffusivity (as compared to arsenic or phosphorus) so that any redistribution of the implanted profiles caused by the electron beam annealing could be examined easily under worst case conditions. Samples were isochronally annealed for $15 \mathrm{~s}$ at different power densities and then the change in sheet resistivity measured. As shown in Fig. 3, the reduction of sheet resistivity exhibited a threshold of $\sim 10 \mathrm{~W} / \mathrm{cm}^{2}$, and

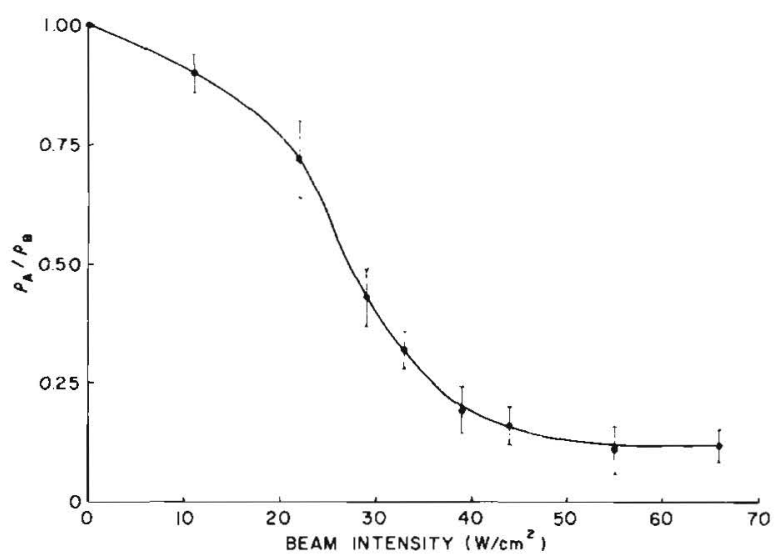

FIG. 3. Reduction in sheet resistivity vs beam power density for a isochronal anneal of $15 \mathrm{~s}$.

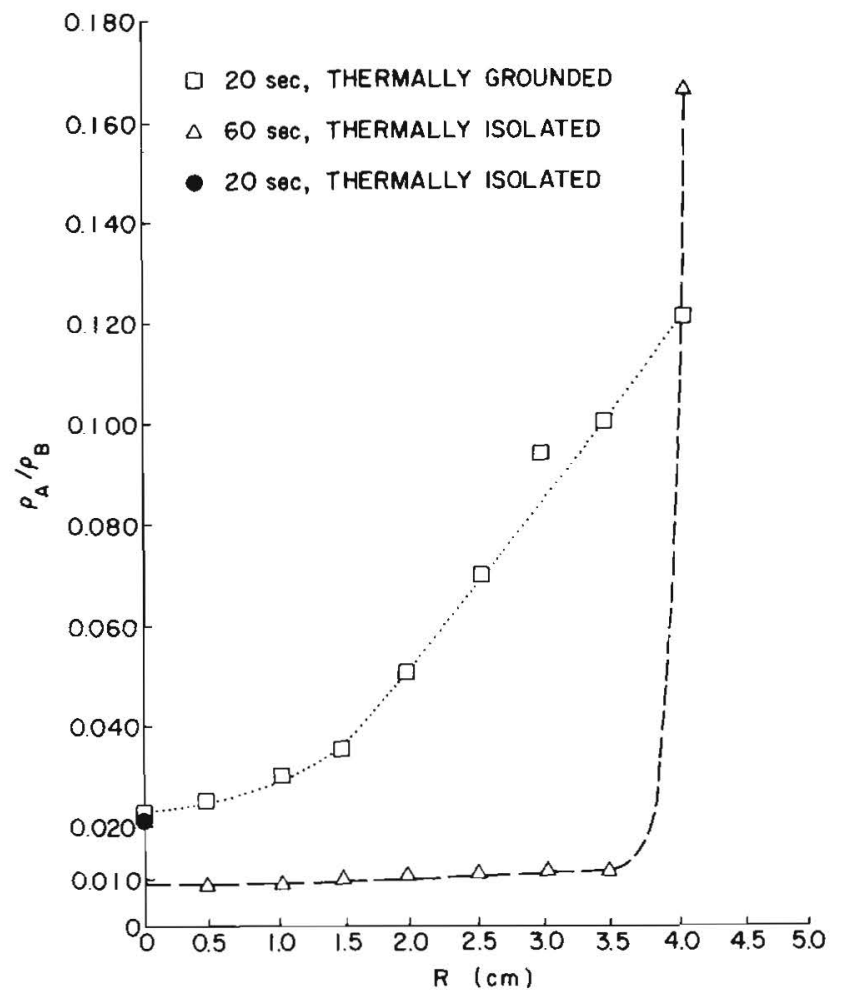

FIG. 4. Sheet resistivity reduction of 10-cm-diam wafers as a function of radius. Wafers were annealed at $400-\mathrm{mA}$ beam current in 1 Torr for $20 \mathrm{~s}$ (upper curve) and $60 \mathrm{~s}$ (lower curve). Single data point is for thermally isolated sample annealed (same condition as upper curve) in center of beam.

reached a 10 times reduction in sheet resistivity at a total energy input of $\sim 50 \mathrm{~W} / \mathrm{cm}^{2}$. Exposures at higher beam powers did not significantly reduce the resistivity below this value. However, longer exposure times and thermal isolation of the wafer can further reduce the resistivity as discussed below and shown in Fig. 4.

The implanted profiles were then examined using secondary ion mass spectrometry (SIMS) in a Cameca IMS-3F with an oxygen primary beam. The samples were sputter profiled using identical conditions and the resulting sputter

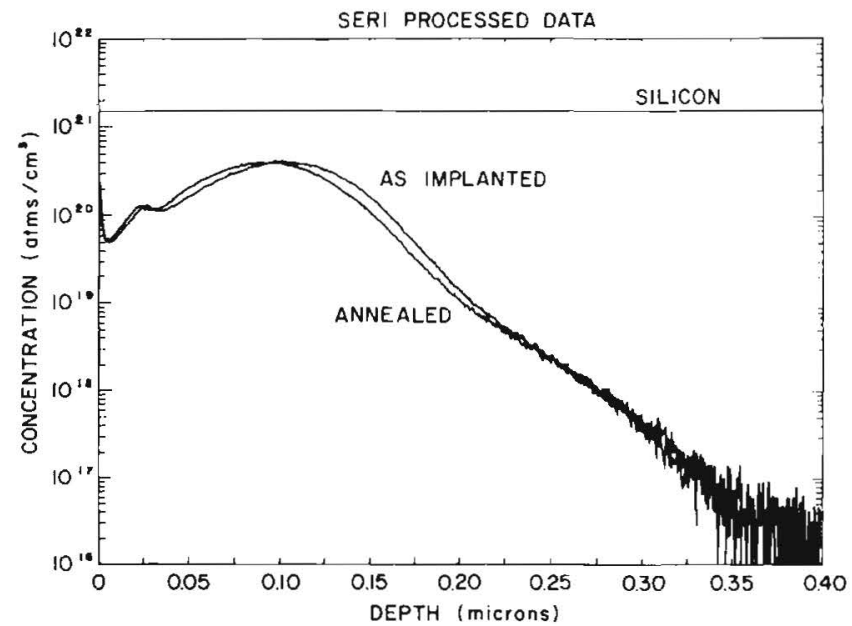

FIG. 5. SIMS depth profiles for as-implanted boron dopant profiles and the electron beam annealed sample, showing minimal, if any, dopant redistribution. Annealing conditions were 15 -s exposure at 1 Torr and 450-mA beam current at a beam voltage of $4 \mathrm{kV}$. 
craters were measured by profilimetry. The comparison between the as-implanted profiles to the annealed profile is shown in Fig. 5 and it is clear from this figure that the electron beam induces minimal dopant redistribution, as compared to a furnace anneal. $^{\text {? }}$

The quality and uniformity of electron beam annealing of large areas were determined by examination of sheet resistivity reduction as a function of radius on $10-\mathrm{cm}$-diam wafers. Wafers of the type described above were annealed at 400-mA beam current in 1 Torr of helium for differing times. The upper curve in Fig. 4 shows the normalized resistivity values as a function of radius for a 20 -s exposure of a heat sunk wafer. Attempting an anneal of greater duration in this configuration resulted in shattering of the wafer. This problem was eliminated by thermally (but not electrically) isolating the wafer, and the lower curve of Fig. 4 was obtained from a wafer annealed for $60 \mathrm{~s}$; both process durations still caused warpage of the wafer due to nonuniform heating of the entire wafer. The single data (for $20 \mathrm{~s}$ while thermally isolated) shows that the effect of sample heat sinking on the annealing process is negligible for 20 -s exposure. A more uniform anneal results for longer exposures, and, since impurity diffusion varies as $\sqrt{t}$, minimal dopant redistribution is still expected for a 60 -s anneal.

In summary, we describe a high power, large area, elec- tron beam source operating in the $3-7-\mathrm{kV}$ region which uses a sintered metal-oxide-metal cathode. Beam generation occurs in a helium ambient of $0.1-2$ Torr. The electron gun is of simple design and is spatially scalable to allow processing of $10 \mathrm{~cm}$ diameter or larger wafers. Implant damage from boron ions has been uniformally annealed over a $7-\mathrm{cm}$-diam area using this source without a significantly disturbing the original implantation profile.

The authors wish to thank Bill Ritchie, Mike Gulett, and Dr. Paul Sullivan of NCR Microelectronics, Fort Collins, for supplying the boron implantation samples used in this work. One author (C.A.M.) acknowledges the support of Colromm, Inc. This work was supported by the Office of Naval Research, the Air Force Office of Scientific Research, and ASM America.

'T. O. Sedgwick, J. Electron Chem. Soc. 130, 484 (1983).

${ }^{2}$ J. L. Regolini, J. F. Gibbons, T. W. Sigmon, R. F. W. Pease, T. J. Magee, and J. Peng, Appl. Phys. Lett. 34, 410 (1979).

${ }^{3}$ J. A. Knapp and S. T. Picraux, Appl. Phys. Lett. 38, 873 (1981).

${ }^{4}$ A. C. Greenwald, A. R. Kirkpatrick, R. G. Little, and J. A. Minnucci, J. Appl. Phys. 50, 783 (1979).

5N. J. Ianno, J. T. Verdeyen, S. S. Chan, and B. G. Streetman, Appl. Phys. Lett. 39, 622 (1981).

'J. J. Rocca, J. D. Meyer, Z. Yu, M. Farrell, and G. J. Collins, Appl. Phys. Lett. 41, $811(1982)$.

${ }^{7}$ T. O. Yep, R. T. Fulks, and R. A. Powell, Appl. Phys. Lett. 38, 162 (1981). 